\title{
CME: Neurology (131836): self-assessment questionnaire
}

\author{
Edited by Philip Smith, Linford Fernandes and Tahseen A Chowdhury
}

\section{SAQs and answers are ONLINE for RCP fellows and collegiate members}

\section{Format}

Candidates are asked to choose the best answer from the five possible answers. This best of five format is used in many medical examinations; however, the questions are not intended to be representative of those used in the MRCP(UK) Part 1 or Part 2 Written Examinations.

\section{The answering process}

1 Go to www.rcplondon.ac.uk/SAQ

2 Log on using your usual RCP username and password

3 Select the relevant $\mathrm{CME}$ question paper

4 Answer all 10 questions by selecting the best answer from the options provided

5 Once you have answered all the questions, click on Submit

\section{Registering your external CPD credits}

Carrying out this activity allows you to claim two external CPD credits. These will be automatically transferred to your CPD diary, where you can review the activity and claim your points.

1. A 28-year-old woman, previously well, presented with numbness in her right leg with a sensory level up to the level of the umbilicus. A partial myelitis was suspected. Magnetic resonance imaging (MRI) of the brain showed three T2 periventricular lesions and one spinal thoracic lesion. There were no enhancing lesions.

What would be the most appropriate next test to establish the diagnosis?

(a) Lumbar puncture and serum sample for oligoclonal bands.

(b) Repeat MRI after 3 months.

(c) Serum aquaporin-4 antibody.

(d) Serum myelin oligodendrocyte glycoprotein.

(e) Visual evoked potentials.
2. Which of the following clinical presentations is atypical for a first presentation of multiple sclerosis?
(a) Bilateral internuclear ophthalmoplegia.
(b) Bilateral optic neuritis.
(c) Isolated sixth nerve palsy.
(d) Transverse myelitis.
(e) Unilateral optic neuritis.

3. A 38-year-old woman with relapsing-remitting multiple sclerosis reported that her walking had become gradually more difficult and she could not walk as far as she used to a couple of years ago. Her walking distance had reduced to about $\mathbf{2 0 0}$ metres before she needed to stop and rest. She was taking a disease modifying treatment and had been treated with methylprednisolone 6 months before, but her symptoms had not improved at all.

What is the best description of her likely disease course?
(a) Primary progressive.
(b) Progressive relapsing.
(c) Relapsing-remitting, active.
(d) Relapsing-remitting, not active.
(e) Secondary progressive.

4. A 22-year-old woman presented with headache and confusion. She had been well until 3 weeks before, when she had some transient coryzal symptoms. MRI of the brain showed multiple lesions in the cerebral white matter, thalami and basal ganglia with some contrast enhancement. Cerebrospinal fluid analysis showed 38 white blood cells per $\mu \mathrm{L}$ ( $100 \%$ lymphocytes). Oligoclonal bands were negative.

\section{What is the most likely diagnosis?}

(a) Acute disseminated encephalomyelitis.

(b) Multiple sclerosis.

(c) Myelin oligodendrocyte glycoprotein antibody disease.

(d) Neuro-Behçet disease.

(e) Neuromyelitis optica spectrum disorder. 
5. A 72-year-old man presented with a 3-month history of difficulties with gradually worsening memory and unusual behaviour, including emotional lability. His family had noticed some jerky movements of his left arm and face on a few occasions over the preceding 3 weeks. On examination, he was alert but distracted and his minimental state examination (MMSE) score was 9/30. MRI of the brain showed age-appropriate cerebral atrophy with T2 signal hyperintensity in the right medial temporal lobe. Cerebrospinal fluid showed white cell count of 8 per $\mu \mathrm{L}$ (normal range $<5$ ) and protein of $0.7 \mathrm{~g} / \mathrm{L}$ (normal $<0.5$ ) with normal plasma:CSF glucose ratio.

\section{What antibody is most likely associated with this presentation?}
(a) AMPAR.
(b) CASPR2.
(c) $G_{A B A} R$.
(d) LGI1.
(e) NMDA-R.

6. A 23-year-old woman was referred from the psychiatric hospital for assessment of suspected seizures and hyperthermia. She had been an inpatient there for 6 weeks receiving neuroleptic medications to treat a first episode of psychosis. On examination, she was drowsy, with writhing movements of her left hand, and repeatedly protruded her tongue. Her temperature was $38.2^{\circ} \mathrm{C}$ and heart rate was 135 beats per minute. MRI of the brain was normal and CSF showed white cell count of 35 per $\mu \mathrm{L}$ (normal range $<5$ ) and protein of $0.4 \mathrm{~g} / \mathrm{L}$ (normal range $<0.5$ ). Computed tomography (CT) of the chest, abdomen and pelvis showed an ovarian mass.

\section{What is the most likely diagnosis?}

(a) Juvenile myoclonic epilepsy.

(b) LGI1 encephalitis.

(c) Neuroleptic malignant syndrome.

(d) NMDA-R encephalitis.

(e) Tardive dyskinesia.

7. A 52-year-old woman, previously well, presented to the emergency department with a single generalised tonicclonic seizure, and a 4-day history of flu-like illness and increasing drowsiness. Her family reported that she had been agitated and aggressive when rousable. On examination, her Glasgow coma scale (GCS) score was 10 (E3, V2, M5), and she was moving all four limbs. CT of the head showed subtle diffuse hypodensity in bilateral medial temporal lobes of uncertain cause, more prominent on the left.

\section{What is the most appropriate initial management?}
(a) Intravenous aciclovir.
(b) Intravenous alteplase.
(c) Intravenous corticosteroid.
(d) Intravenous immunoglobulin.
(e) Plasma exchange.

8. A 25-year-old woman with idiopathic intracranial hypertension was prescribed acetazolamide. The doctor explained the possible side effects and highlighted the most common of these.

What side effect does the doctor most likely highlight?
(a) Bone marrow disorders.
(b) Confusion.
(c) Paraesthesiae.
(d) Thrombocytopenia.
(e) Tinnitus.

9. A 20-year-old woman has idiopathic intracranial hypertension associated with obesity. She agreed to start topiramate and the neurologist explained to her its possible side effects, highlighting one particular side effect as the most common.

\section{What side effect does the neurologist most likely highlight?}
(a) Glaucoma.
(b) Raynaud's phenomenon.
(c) Sexual dysfunction.
(d) Tingling.
(e) Weight gain.

10. A 62-year-old man attended for review of his probable Parkinson's disease. The doctor reviewed his symptoms and investigations and noted a particular clinical feature that is more suggestive of an alternative diagnosis.

\section{What feature does the doctor most likely highlight?}

(a) Abnormal MIBG SPECT imaging.

(b) Good response to levodopa therapy.

(c) Levodopa-induced dyskinesia

(d) Olfactory loss.

(e) Recurrent falls within 3 years of onset.

CME: Renal medicine SAQ Answers to the CME SAQ published in Clinical Medicine in March 2020
Q1 Q2
Q3
Q4 Q5 Q6
Q7 Q8
Q9 Q10
(e) (d) (b)
(b) (b)
(b) (b)
(a) (c) 